\title{
Title of the Article: A Rare Case Report of Diffuse Large B- Cell Lymphoma of Bone with Multicentric Involvement
}

\author{
Naveen Mittal* \\ Department of orthopaedics, Government Medical College Hospital, India
}

*Corresponding author: Naveen Mittal, Department of orthopaedics, Government Medical College Hospital, Sector-32, Chandigarh, India Submission: 眥 February 07, 2018; Published: 温 March 14, 2018

\begin{abstract}
Primary bone lymphomas (PBL) are rare, even though secondary involvement of the bone marrow is a common event in systemic lymphomas. Most PBL are Primary Bone Diffuse Large B-Cell Lymphomas (PBDLBCL). In the present case report, a 58 year female presented with long term pain in the left thigh along with palpable subcutaneous masses in the head. The MRI done in the periphery reported it as a cystic lesion and was advised extended curettage. We reviewed the case and carried out CT pelvis with left thigh and CT head to see for bony involvement which showed involvement of the sacrum, femur and parietal bones. The lesions were biopsied subjected to histopathology and immunohistochemistry which confirmed the diagnosis .The patient is presently on CHOP regime highlighting the large frequency with which such cases are misdiagnosed leading to progression of the disease.
\end{abstract}

Keywords: Primary bone diffuse large B-cell lymphoma (PBDLBCL); Misdiagnosis; Immuno histo chemistry; CHOP regime

\section{Introduction}

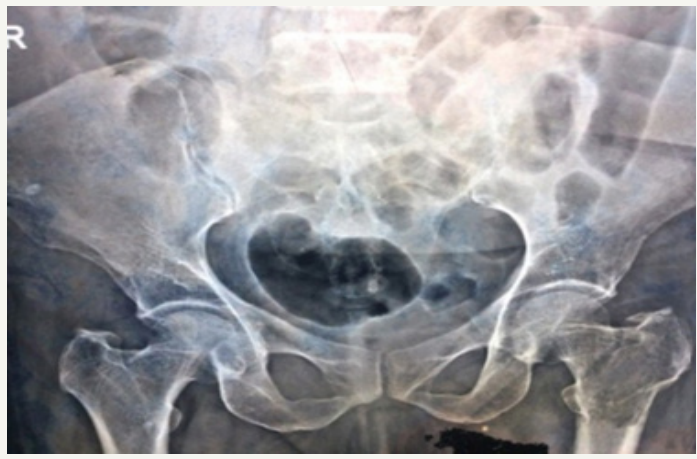

Figure 1: Radiograph showing an osteolytic lesion in the proximal femur on AP view of the pelvis.

Primary Bone Lymphomas(PBL) are rare, accounting for less than $1 \%$ of all malignant lymphomas, $7 \%$ of malignant bone tumours and $4-5 \%$ of extra-nodal lymphomas. Most are PBDLBCL with a rare occurrence of follicular, marginal zone, anaplastic large cell, Hodgkin and T- cell lymphomas [1]. The patient presents with bone pain, palpable mass, fractures or neurological symptoms. Differential diagnosis of PBDLBCL includes primary bone sarcoma, multiple myeloma, leukemic infiltrate, chronic osteomyelitis, Ewing sarcoma, metastatic sarcomas and carcinoma [2]. It is a very rare diagnosis having excellent prognosis for recovery. We present a case report of PBDLBCL with multicentric involvement.

\section{Case History}

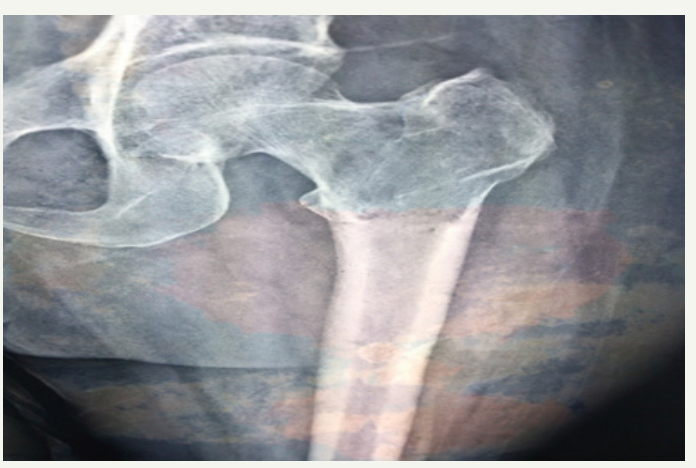

Figure 2: Radiograph lateral view thigh highlighting the lesion.

A 58 year old female presented with pain in the left thigh and pelvis. She was originally radiographed (Figure $1 \& 2$ ) and was managed conservatively in the periphery. The radiographed of the skull showed no involvement of skull bones (Figure 3).Then the MRI of the femur and pelvis was done which reported it is as a cystic lesion and was advised extended curettage (Figure $4 \& 5$ ). No treatment was done. Two months after this the patient presented to us with greater pain and a pathological fracture left trochanteric region. The patient also had palpable subcutaneous masses in the 
head. We reviewed the MRI and did CECT pelvis and CECT head. CECT pelvis showed lytic expansile destruction of both the sacral ala with soft tissue masses extending into spinal canal causing compression of the cal sac in addition to the original lesion in the neck and trochanteric region with fracture (Figure 6). CECT head showed multiple lytic areas with formation of soft tissue masses in extra-calvirial and intra cranial locations (Figure 7). The brain parenchyma was normal. We did biopsy of the trochanteric region and nodule from scalp. Both the reports showed large tumour cells having round, irregular cleaved nuclei with 1-2 prominent nucleoli and moderate amount of cell cytoplasm with frequent mitotic figures (Figure 8). On immunohistochemistry were positive for LCA and CD-20 suggesting diffuse large B- cell lymphoma (Figure 9). The patient was then started on CHOP regime.

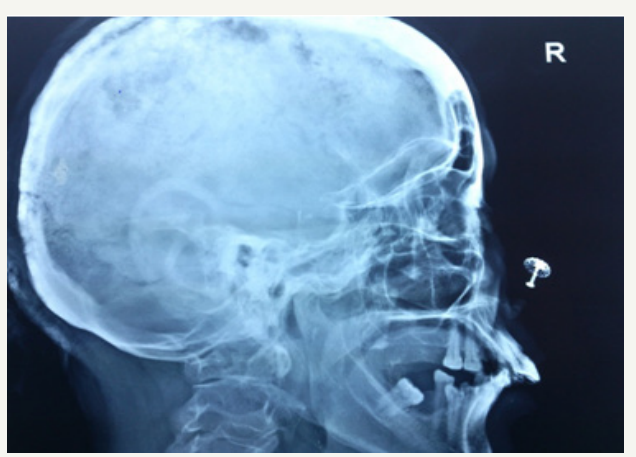

Figure 3: Radiograph skull showing no involvement of the skull bones.

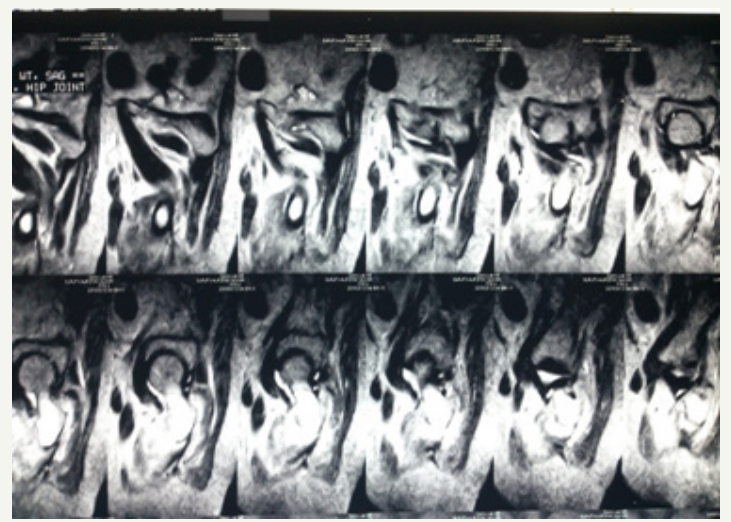

Figure 4: MRI sagittal sections showing extent of the lesion.

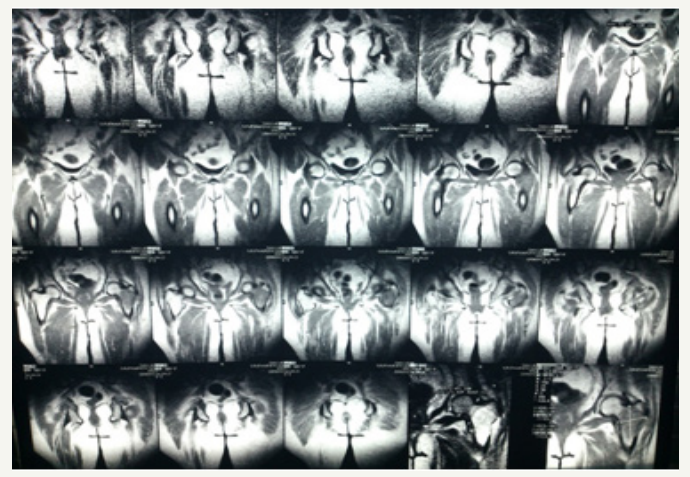

Figure 5: MRI coronal sections highlighting the lesion.

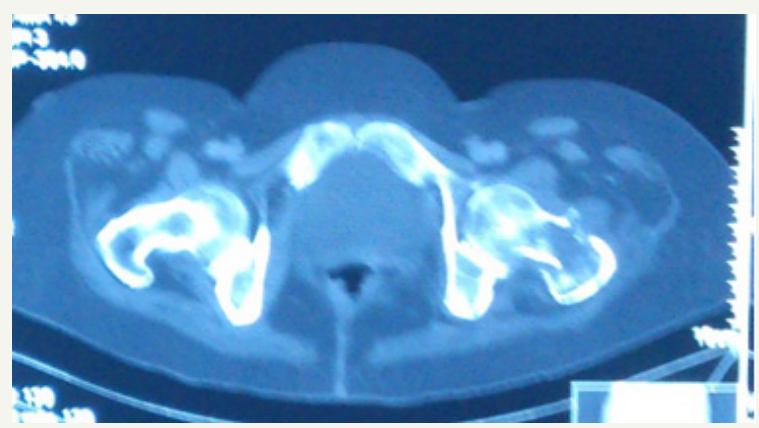

Figure 6: CT showing the pathological fracture with the lesion.

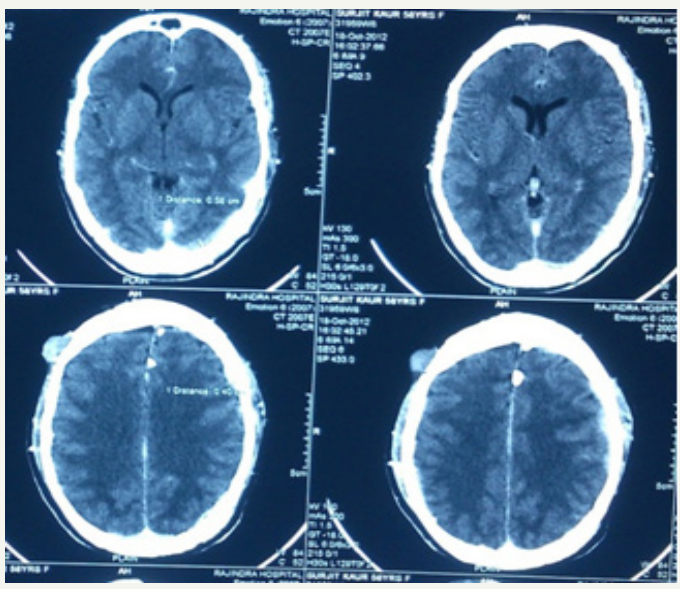

Figure 7: CT skull showing no involvement of the extracalvarial or intracalvarial structures.

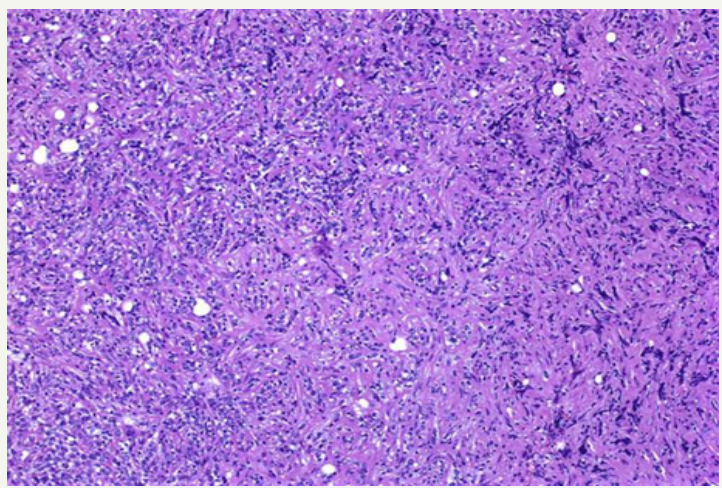

Figure 8: Tumour cells having round, irregular cleaved nuclei with prominent nucleoli and moderate amount of cell cytoplasm with frequent mitotic figures suggestive of round cell tumour.

\section{Discussion}

Primary Bone Lymphoma was first reported by Oberling [3]. Parker and Jackson reported a PBL series in 1939 under the designation reticulum cell sarcoma of bone, establishing PBL as a distinct entity [4]. According to 2002 World Health Organization classification of tumours of soft tissue and bone, the criteria for a diagnosis of PBL are

a. A single skeletal tumour without regional lymph node involvement. 
b. Multiple bone lesions without visceral or lymph node involvement [5].

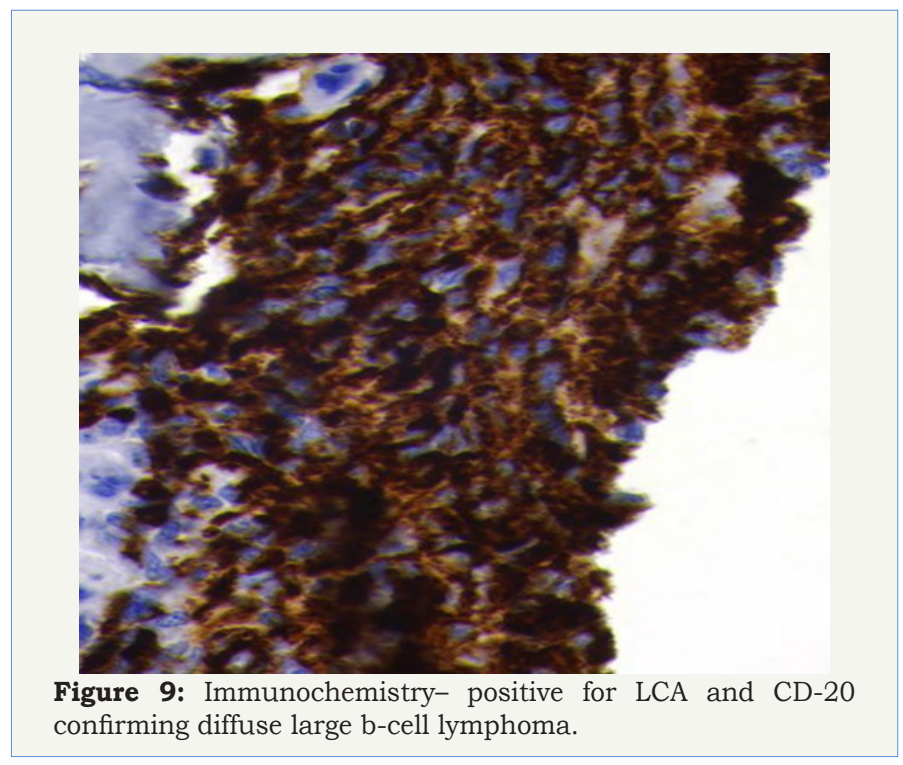

In terms of anatomic involvement the most common site arefemur(27\%), pelvis(15\%), tibia/ fibula(13\%), polyostotic(13\%), humerus(12\%), spine(9\%), skull(1\%) etc as reported by Beal et al. with a male-female ratio of 1.5:1 and a median age of 48 years [6]. Radiologically bony lesions may be extensive, destructive or infiltrative. Extensive lesions involve $25-50 \%$ of affected bone, sometimes entire bone. Destructive lesions are radiolucent, mottled, patchy, moth eaten and sometimes the outline of the bone is completely lost. Infiltrative or permeative lesions have poorly defined interface with normal bone, $50 \%$ have a mixture with small areas of sclerosis. Nearly all variants destroy cortical bone and 25\% thicken the cortex. Often large, obvious soft-tissue extension may be present along with bony lesion [7]. Diagnosis is established by biopsy. The tumor cells are positive for CD45, CD20, CD79a, LCA and negative for CD3 and CD5 [8].

Traditionally, the treatment has been combination of chemotherapy and radiotherapy. But over the last two decades, chemotherapy (CHOP based) has become the standard treatment with or without consolidative irradiation. The introduction of rituximab has been accompanied by a remarkable improvement (3-year PFS 88\% versus 52\%) [9].

Role of radiation in advanced stage PBL is controversial. In a study by Ramadan et al. [10] patients with advanced-stage disease who received chemotherapy plus irradiation actually had a poorer outcome compared with those who received chemotherapy alone (10-year OS were $25 \%$ and $56 \%$, respectively). However, this difference must be very cautiously interpreted because the decision to use irradiation was individualized. Surgery is only indicated for pathologic fractures.

We want to highlight here the rare conditions like PBDLBCL which are not thought of. Diagnosis such as this are not thought of in the periphery. There is a very high frequency in misdiagnosis. Patients are often started on treatment just on the basis of biased MRI reports considering the most common diagnosis. Instead the patient should be thoroughly investigated according to a protocol and managed. If this patient had not reported to us before it was too late, the prognosis would have been worse. The treatment often is simple and known to everyone but establishing the diagnosis requires a careful supervision. Immunohistochemistry is highly important for establishing the correct diagnosis which is often overseen. Thus, primary lymphoma of bone should be considered in the differential diagnosis of bone tumors. We did not use rituximab, but addition may have benefited.

\section{References}

1. Desai S, Jambhekar NA, Soman CS, Advani SH (1991) Primary lymphoma of bone:a clinicopathologic study of 25 cases over 10 years. J Surg Oncol 46(4): 265-269.

2. Fidias P, Spiro I, Sobczak ML, Nielsen GP, Ruffolo EF, et al. (1999) Longterm results of combined modality therapy in primary bone lymphomas. Int J Radiat Oncol Biol Phys 45(5): 1213-1218.

3. OberlingC(1928) Les reticulosarcomesetles reticuloendotheliosarcomes de la osseuse. Bull Assoc Fr Etude Cancer 17: 259-296

4. Parker F, Jackson H (1939) Primary reticulum cell sarcoma of bone. Modern radiation oncology 68: 45-53

5. Unni KK, Hogendroon PCW (2002) Malignant lymphoma. In: Fletcher CDM, Unni KK, Mertens F (Eds.), Pathology and Genetics of Tumors of Soft Tissue and Bone, IARC Press World Health Organization Classification of Tumours, France.

6. Beal K, Allen L, Yahalom J (2006) Primary bone lymphoma: treatment results and prognostic factors with long term follow up 82 patients. Cancer 106(12): 2652-2656.

7. Heyning FH, Hogendoorn PC, Kramer MH, Hermans J, Kluin Nelemans JC, et al. (1999) Primary non-Hodgkin's lymphoma of bone: A clinicopathological investigation of 60 cases. Leukemia 13(12): 20942098.

8. Hans CP, Weisenburg DD, Greiner T, Gascoyne RD, Delabie J, et al. (2004) Confirmation of molecular classification of diffuse large B-cell lymphoma by immunohistochemistry using a tissue microarray. Blood 103(1): 275-282.

9. Baar J, Burkes RL, Bell R, Blackstein ME, Fernandes B, et al. (1994) Primary non-Hodgkin's lymphoma of bone: A clinicopathological study. Cancer 73(4): 1194-1199.

10. Ramadan KM, Shenkier T, Sehn LH, Gascoyne RD, Connors JM (2007) A clinicopathological retrospective study of 131 patients with primary bone lymphoma: A population-based study of successively treated cohorts from the British Columbia Cancer Agency. Ann Oncol 18(1): 129-135. 
Creative Commons Attribution 4.0 International License

For possible submissions Click Here

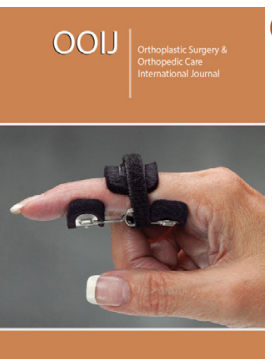

Orthoplastic Surgery \& Orthopedic Care International Journal

\section{Benefits of Publishing with us}

- High-level peer review and editorial services

- Freely accessible online immediately upon publication

- Authors retain the copyright to their work

- Licensing it under a Creative Commons license

- Visibility through different online platforms 